\title{
Meta
}

Journal des traducteurs

Translators' Journal

\section{Liste des relecteurs 2016}

Volume 61, numéro 3, décembre 2016

URI : https://id.erudit.org/iderudit/1039214ar

DOI : https://doi.org/10.7202/1039214ar

Aller au sommaire du numéro

Éditeur(s)

Les Presses de l’Université de Montréal

ISSN

0026-0452 (imprimé)

1492-1421 (numérique)

Découvrir la revue

Citer ce document

(2016). Liste des relecteurs 2016. Meta, 61(3), 501-504.

https://doi.org/10.7202/1039214ar

Ce document est protégé par la loi sur le droit d'auteur. L’utilisation des services d'Érudit (y compris la reproduction) est assujettie à sa politique d'utilisation que vous pouvez consulter en ligne.

https://apropos.erudit.org/fr/usagers/politique-dutilisation/
Cet article est diffusé et préservé par Érudit.

Érudit est un consortium interuniversitaire sans but lucratif composé de l’Université de Montréal, l'Université Laval et l'Université du Québec à Montréal. Il a pour mission la promotion et la valorisation de la recherche. https://www.erudit.org/fr/ 


\section{Liste des relecteurs 2016}

Nous remercions chaleureusement toutes celles et ceux qui ont donné de leur temps et qui ont contribué à la revue. Toute omission est involontaire de notre part, veuillez nous la signaler.

Esperanza Alarcón Navio (Universidad de Granada)

Icíar Alonso Araguás (Universidad de Salamanca)

Ruth Amossy (Tel Aviv University)

Gemma Andújar Moreno (Universitat Pompeu Fabra)

Dimitris Asimakoulas (University of Surrey)

Alexandra Assis Rosa (Universidade de Lisboa)

Jesús Baigorri-Jalón (Universidad de Salamanca)

Christian Balliu (Haute École de Bruxelles)

Paul Bandia (Concordia University)

Marie-Alice Belle (Université de Montréal)

Mario Bisiada (Universitat Pompeu Fabra)

Pier-Pascale Boulanger (Concordia University)

Lynne Bowker (Université d'Ottawa)

Louise Brunette (Université du Québec en Outaouais)

Helena Buffery (University College Cork)

Hélène Buzelin (Université de Montréal)

Philippe Caignon (Concordia University)

Catalina Calafat Ripoll (Universitat de les Illes Balears)

Nayelli Maria Castro-Ramirez (University of Massachusetts Boston)

Chirine Chamsine (Université de Montréal)

Marc Charron (Université d'Ottawa)

Frederic Chaume Varela (Universitat Jaume I)

Sungdai Cho (Binghampton University)

Donna Chu (Chinese University of Hong Kong)

Gloria Corpas Pastor (Universidad de Málaga)

Nicole Côté (Université de Sherbrooke)

Michele De Gioia (Università degli Studi di Padova)

Irene de Higes Andino (Universitat Jaume I)

Paula de Santiago (Universidad de Valladolid)

Dirk Delabastita (Université de Namur)

Jorge Díaz-Cintas (University College London)

Christine Durieux (Université de Caen Basse-Normandie)

Álvaro Echeverri (Université de Montréal) 
Izaskun Elorza (Universidad de Salamanca)

Ahu Selin Erkul Yağcı (Ege Üniversitesi)

Carlo Eugeni (Università degli Studi di Macerata)

Manuela Fernández Sánchez (Universidad de Granada)

Marco A. Fiola (Ryerson University)

Peter Flynn (Katholieke Universiteit Leuven)

Lydia Fossa (Pontificia Universidad Católica del Perú)

Javier Franco Aixelá (Universitat d'Alacant)

Maria Freddi (Università degli Studi di Pavia)

Judit Freixa Aymerich (Universitat Pompeu Fabra)

Nicolas Froeliger (Université Paris 7 - Diderot)

Chantal Gagnon (Université de Montréal)

Silvia Gamero Pérez (Universitat Jaume I)

Fang Gao (Nanjing University)

Olalla García Becerra (Universidad de Granada)

Nizar Ghazzawi (Université de Montréal)

Anna Ghiglione (Université de Montréal)

Daniel Gile (Université Paris 3 - Sorbonne Nouvelle)

Juan Carlos Godenzzi (Université du Québec à Montréal)

José Luiz Gonçalves (Universidade Federal de Ouro Preto)

Rebecca Gould (University of Bristol)

Zélie Guével (Université Laval)

Hugh Hazelton (Concordia University)

Lance Hewson (Université de Genève)

Isaac Hui (Lingnan University)

John Humbley (Université Paris 7 - Diderot)

Paula Igareda (Universitat Autònoma de Barcelona)

Catalina Iliescu Gheorghiu (Universitat d'Alacant)

Judith Inggs (University of the Witwatersrand Johannesburg)

Esmaeil Kalantari (Université de Montréal)

Daejin Kim (Seoul National University of Science and Technology)

Sara Kippur (Trinity College)

Donald Kiraly (Johannes-Gutenberg-Universität Mainz)

Louise Ladouceur (University of Alberta)

Christian Lagarde (Université de Perpignan)

Gillian Lane-Mercier (McGill University)

Elisabeth Lavault-Olléon (Université Stendhal - Grenoble 3)

Sara Laviosa (Università degli Studi di Bari Aldo Moro)

Judith Lavoie (Université de Montréal)

Élisabeth Le (University of Alberta)

Émeline Lecuit (École supérieure du professorat et de l'éducation Lille-Nord-de-

France)

Benoit Léger (Concordia University)

Wenjing Li (The Hong Kong Polytechnic University)

Defeng Li (University of Macau)

Bo Li (City University of Hong Kong)

Christy (Fung-ming) Liu (The Hong Kong Institute of Education) 
Kazem Lotfipour-Saedi (chercheur indépendant)

Fransiska Louwagie (University of Leicester)

Georgiana Lungu-Badea (Universitatea de Vest din Timişoara)

Oumarou Mal Mazou (Université de Liège)

Kirsten Malmkjaer (University of Leicester)

Carme Mangiron Hevia (Universitat Autònoma de Barcelona)

Danièle Marcoux (Concordia University)

Elizabeth Marshman (Université d'Ottawa)

Marta Mateo Martínez-Bartolomé (Universidad de Oviedo)

Christopher Mellinger (Walsh University)

Jun Miao (Institut national des langues et civilisations orientales)

Holly Mikkelson (Middlebury College)

Helen Julia Minors (Kingston University London)

Sylvie Monjean-Decaudin (Université de Cergy-Pontoise)

Brian Mossop (York University)

Alexis Nouss (Université d'Aix-Marseille)

Christian Olalla Soler (Universitat Autònoma de Barcelona)

Emilio Ortega Arjonilla (Universidad de Málaga)

María Teresa Ortego-Antón (Universidad de Valladolid)

Gilles Ouvrard (Hankuk University of Foreign Studies)

Outi Paloposki (Turun yliopisto)

Réal Paquette (Université de Montréal)

Maria Pavesi (Università degli Studi di Pavia)

Franz Pöchhacker (Universität Wien)

Ayşenaz Postalcıŏlu (Boğaziçi Üniversitesi)

Isabelle Poulin (Université Bordeaux-Montaigne)

Anthony David Pym (Universitat Rovira i Virgili)

Christine Raguet (Université Paris 3 - Sorbonne Nouvelle)

Sathya Rao (University of Alberta)

Alessandra Riccardi (Università degli Studi di Trieste)

Françoise Rigat (Università della Valle d'Aosta)

Isabelle Robert (Universiteit Antwerpen)

Roda P. Roberts (Université d'Ottawa)

Margaret Rogers (University of Surrey)

Myriam Salama-Carr (The University of Manchester)

Elena Sánchez Trigo (Universidade de Vigo)

John Douglas Sanderson (Universitat d'Alacant)

Julio César Santoyo Mediavilla (Universidad de León)

Elena Sanz-Ortega (University of Edinburgh)

Candace Séguinot (York University)

Adriana Serban (Université Paul-Valéry Montpellier 3)

Annie Shao (Southwestern University of Finance and Economics)

James St. André (Chinese University of Hong Kong)

Bernd Stefanink (Universidade Federal de Santa Catarina)

Madeleine Stratford (Université du Québec en Outaouais)

Myriam Suchet (Université Paris 3 - Sorbonne Nouvelle)

Yifeng Sun (Lingnan University) 
Kayoko Takeda (Rikkyo University)

Zaixi Tan (Hong Kong Baptist University)

Luciana Sabina Tcaciuc (Banque centrale européenne)

Teresa Tomaszkiewicz (Uniwersytet im. Adama Mickiewicza)

Anne Tomiche (Université Paris 4 - Paris-Sorbonne)

Cynthia Tsui (Hong Kong Baptist University)

Christoph Unger (Norges Teknisk-Naturvitenskapelige Universitet)

Roberto Antonio Valdeón (Universidad de Oviedo)

Egan Valentine (Université du Québec à Trois-Rivières)

Carmen Valero Garcés (Universidad de Alcalá)

Hugo Vandal-Sirois (Université de Montréal)

Gudrun Vanderbauwhede (Université de Mons)

Daniel Steve Villarreal (National Defense Medical Center)

Jane Elisabeth Wilhelm (Université Sorbonne Nouvelle - Paris 3)

Malcolm Williams (Université d'Ottawa)

Michaela Wolf (Karl-Franzens-Universität Graz)

Dorothy Wong (Lingnan University)

Christine York (Concordia University)

Federico Zanettin (Università degli Studi di Perugia)

Junfeng Zhang (Central China Normal University)

Florence Zhang (Université Paris 7 - Diderot) 